\title{
Transcranial sonography findings related to depression in parkinsonian disorders: cross-sectional study in 126 patients
}

Angela E.P. Bouwmans, Wim E.J. Weber, Albert F.G. Leentjens, Werner H. Mess

Background: Transcranial sonography (TCS) has emerged as a potential diagnostic tool for Parkinson's disease. Recent research has suggested that abnormal echogenicity of substantia nigra, raphe nuclei and third ventricle is associated with increased risk of depression among these patients. We sought to reproduce these findings in an ongoing larger study of patients with parkinsonian syndromes.

Methods: 126 patients with parkinsonian symptoms underwent the Hamilton Depression Scale, and TCS of the substantia nigra (SN) $(n=126)$, the raphe nuclei $(R N)(n=80)$ and the third ventricle $(n=57)$. We then calculated correlation between depression and hyperechogenic SN, hypo-echogenic RN and a wider third ventricle.

Results: In patients with PD we found no significant difference of the SN between nondepressed and depressed patients ( $46 \%$ vs. $22 \% ; p=0.18$ ). Non-depressed patients with other parkinsonisms more often had hyperechogenicity of the SN than depressed patients ( $51 \%$ vs. $0 \% ; p=0.01$ ). We found no relation between depression and the echogenicity of the RN or the width of the third ventricle.

Conclusions: In patients with parkinsonian syndromes we found no association between depression and hyper-echogenic SN, hypo-echogenic RN or a wider third ventricle, as determined by transcranial sonography. 


\section{Transcranial sonography findings related to}

\section{2 depression in parkinsonian disorders: cross-}

\section{3 sectional study in 126 patients}

4

5

6

E-mail : angelabouwmans@,outlook.com

Tel.: $+31-43 / 3875058$

Fax. : $+31-43 / 3877055$

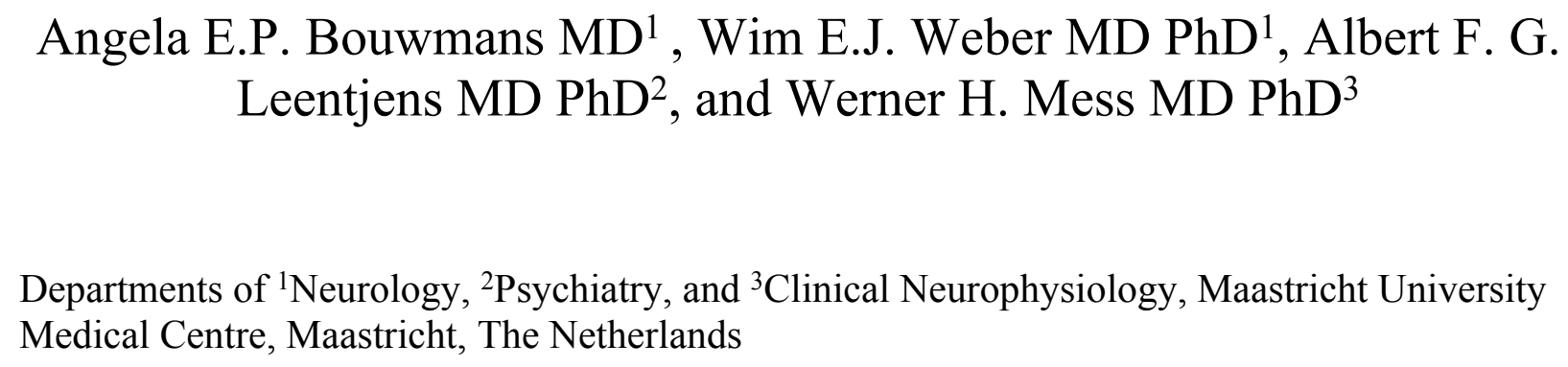

Corresponding author: dr. A.E.P Bouwmans, Dept. of Neurology, Maastricht University Medical Centre, PO Box 5800, 6202 AZ Maastricht, The Netherlands

20 


\section{Abstract}

25 Background Transcranial sonography (TCS) has emerged as a potential diagnostic tool for

26 Parkinson's disease. Recent research has suggested that abnormal echogenicity of substantia

27 nigra, raphe nuclei and third ventricle is associated with increased risk of depression among

28 these patients. We sought to reproduce these findings in an ongoing larger study of patients with

29 parkinsonian syndromes of recent onset.

31 Methods 126 patients with parkinsonian symptoms underwent the Hamilton Depression Scale, 32 and TCS of the substantia nigra $(\mathrm{SN})(\mathrm{n}=126)$, the raphe nuclei $(\mathrm{RN})(\mathrm{n}=80)$ and the third 33 ventricle $(\mathrm{n}=57)$. We then calculated correlation between depression and hyper-echogenic $\mathrm{SN}$, 34 hypo-echogenic RN and a wider third ventricle.

36 Results In patients with PD we found no significant difference in echogenicity of the SN

37 between non-depressed and depressed patients ( $46 \%$ vs. $22 \%$; $=0.18)$. Non-depressed patients

38 with other parkinsonisms more often had hyperechogenicity of the SN than depressed patients

$39(51 \%$ vs. $0 \% ; p=0.01)$. We found no relation between depression and the echogenicity of the

$40 \mathrm{RN}$ or the width of the third ventricle.

42 Conclusions In patients with recent-onset parkinsonian syndromes we found no association

43 between depression and hyper-echogenic SN, hypo-echogenic RN or a wider third ventricle, as 44 determined by transcranial sonography. 
46 Trial registration: ITRSCC NCT00368199 
48

49

50

51

52

53

54

55

56

57

58

59

60

61

62

63

64

65

66

67

68

69

70

71

0

\section{Introduction}

49

Idiopathic Parkinson's disease (PD) is the second most common neurodegenerative disease with a worldwide prevalence of 41 to 1903 per 100,000 (Pringsheim et al. 2014). Diagnosis, especially in the early stages, is difficult, as there is no definitive diagnostic test. Over the last 10 years transcranial sonography (TCS) of the substantia nigra (SN) has emerged as a promising tool in this regard. Numerous ultrasound studies have found that a significant percentage of patients with IPD have a typical enlarged area of echogenicity in the substantia nigra $(\mathrm{SN}+)$, which is thought to be associated with increased iron concentrations (Vlaar et al. 2009).

Although PD is mostly known for its motor symptoms, it has now become clear that non-motor symptoms, such as depression, often contribute to the burden of disease (Reijnders et al. 2008). Depression has a major impact on PD patients: depressed PD patients have worse motor function, more cognitive symptoms, and a lower quality of life (Reijnders et al. 2008; Schrag 2006; Schrag et al. 2010). The pathogenesis of depression in PD is still unknown. Studies have suggested that the serotonergic raphe nuclei (RN) might be involved (Becker et al. 2001; Chagas et al. 2013; Kostic \& Filippi 2011; Leentjens 2004; Palhagen et al. 2008).

67 Sonography researchers have thus investigated the RN in PD patients, and reported that its echogenicity was reduced in depressed PD patients compared to non-depressed PD patients and 9 healthy control subjects (Becker et al. 1997; Berg et al. 1999b; Cho et al. 2011; Stankovic et al. 2015; Zhang et al. 2015). Additionally, SN hyperechogenicity and a wider third ventricle has 71 also been reported to be associated with an increased risk of depression (Krogias et al. 2011; 
72 Walter et al. 2007c; Walter et al. 2010). TCS could be clinically useful, as diagnosing depression

73 in PD patients is difficult (Bouwmans \& Weber 2012; Poewe \& Luginger 1999; Shulman et al.

74 2002).

75

76 We recently finished a prospective cohort study on the diagnostic accuracy of TCS in early

77 parkinsonian patients (Bouwmans et al. 2013), and we used this dataset to explore the association

78 between depressive symptoms and echogenic features of the $\mathrm{SN}, \mathrm{RN}$, and third ventricle in PD

79 patients.

80

81

82 


\section{Patients and methods}

84 Design

85 This study was a cross-sectional study, nested within a prospective cohort study that aimed to

86 test the diagnostic accuracy of TCS of the SN in patients who were referred to a neurologist by

87 their general practitioner (GP) because of recent-onset parkinsonism of unclear origin

88 (Bouwmans et al. 2013) The study protocol was published before patient inclusion started (Vlaar

89 et al. 2007). The main finding of the cohort study was that the diagnostic accuracy of the

90 echogenicity of the SN as a diagnostic test for early PD is not sufficient for routine clinical use.

91 The Institutional Review Board (IRB) of Maastricht University Medical Centre approved the

92 study (MEC 05-228, 4 April 2006), which was registered in the ClinicalTrials.gov database as

93 NCT00368199. Both the TCS and the Hamilton Depression Scale assessments were pre-

94 specified in the published protocol. However, this study of the association between the two was

95 not prespecified, and is thus a posthoc analysis.

97 Patients

98 We considered 283 consecutive patients with parkinsonism of unknown origin, who were

99 referred to the neurology outpatient clinic of Maastricht University Medical Centre, Maastricht

100 and the Orbis Medical Centre, Sittard, the Netherlands (Presently: Zuyderland Medical Centre).

101 Patients who did not consent or those in whom a definite diagnosis could be made at the first

102 visit $(\mathrm{n}=42)$ were excluded from the study. Hence, 241 patients were included. Of these, another

10369 were excluded: 24 patients who upon examination did not present with clear parkinsonian

104 symptoms or who presented with drug-induced parkinsonism, as well as 45 patients $(18.7 \%)$ who

105 did not have a sufficient bone window for an adequate TCS examination (See flowchart). 
107 Measures

108 After signing informed consent, all subjects underwent a structured interview and a neurological

109 examination (Bouwmans et al. 2013; Vlaar et al. 2007). All tests were performed by a physician

110 (Senior resident in neurology) not treating the patient and blinded for information in clinical

111 records. Depressive symptoms were measured with the observer-rated 17 item Hamilton

112 Depression Rating Scale (HAMD). This scale has a good reliability and validity, both in PD

113 patients as well as in the general population. (Leentjens et al. 2000; Schrag et al. 2007). The

114 score range is 0 to 52, with scores of 11 and higher suggesting clinically relevant depressive

115 symptoms. Motor symptoms were measured with the Unified Parkinson's Disease Rating Scale

116 (UPDRS-III) (Movement Disorder Society Task Force on Rating Scales for Parkinson's 2003).

117 Within two weeks of inclusion all patients underwent a TCS at the department of Clinical

118 Neurophysiology of one of the two hospitals. In Maastricht University Medical Center,

119 visualization of the RN was included in the TCS protocol from the start of the study. One year

120 later, measurement of the third ventricle was included as well. In the Orbis Medical Center,

121 Sittard, only the SN was visualized.

122 TCS was performed using a SONOS 5500 system (Philips, Eindhoven, the Netherlands). The

123 examination took place in a darkened room with the patient already lying on the examination

124 table before the investigator entered the room, in order to minimize the risk of identification of a

125 patient's clinical signs. Patient and investigator were instructed not to discuss symptoms or

126 diagnoses.

127 TCS was performed bilaterally through the pre-auricular bone window with a $2-4 \mathrm{MHz}$ phased

128 array transducer. The quality of the bone window was scored as good, moderate or inferior. 
129 . Two different methods were applied for the evaluation of the echointensity of the SN. Firstly,

130 the presence or absence of an obviously visible bilateral hyperechogenic SN was scored

131 (qualitative method). The $\mathrm{SN}$ were scored as hyperechointens, not hyperechointens or

132 inconclusive (= no typical configuration of hyperechointensity or low quality of the temporal

133 bone window). Secondly, the area of an eventually hyperechogenic SN was measured

134 quantitatively (quantitative method). Both the right and left SN were measured from both sides,

135 i.e. both temporal bone windows. After encircling, the area was automatically calculated. A

136 hyperechogenic area of at least $0.2 \mathrm{~cm} 2$ was classified as characteristic for PD.. This was only

137 performed when the hyperechogenicity was located within the anatomical distribution of the SN,

138 meaning that it showed a typical oblique stripe-shaped configuration. Both the right and left SN

139 were measured from both sides.

140 The RN were identified if they met the criteria of an anatomic structure equally echo-intense to

141 the red nucleus and localized in the transverse plane of the midbrain with a length extending

142 from anterior to posterior, not interrupted. Echogenicity of the RN was rated using a visual

143 scoring system resulting in a semi-quantitative assessment. We scored the RN as hypo-echogenic

144 (RN-) when this structure had a reduced echogenicity compared to the surrounding brain

145 structures or when the anatomic structure was interrupted. We scored the RN as hyperechogenic

$146(\mathrm{RN}+)$ on the TCS when it showed as an uninterrupted relatively echo-intense structure. The

147 patient was scanned from both sides because of the bone window variability in quality of

148 visualization of the $\mathrm{RN}$ from right to left. We used the best possible result, so if the RN was

149 absent on one side, but visible at the other side, it was scored as hyperechogenic.

150 The transverse diameter of the third ventricle was measured from both sides on a standardized

151 diencephalic examination plane. 
153 Two years after inclusion, patients were re-examined by two movement disorder neurologists to

154 obtain a final clinical diagnosis, using the official diagnostic criteria for the several parkinsonian

155 disorders (Gilman et al. 2008; Hughes et al. 1992; Litvan et al. 1996; Litvan et al. 1999; McKeith

156 et al. 2005), which served as a gold standard for our study. These investigators were blinded for

157 all test results, and none of the neurologists had seen the patient before. They were asked to

158 interview and examine the patient, as they would normally do during a routine neurologic

159 consultation. The neurologists filled in the same standard form as had been done by the including

160 investigator during the first visit of the patient, which included, among others, the Unified

161 Parkinson's Disease Rating Scale (UPDRS)-III score. Afterwards the neurologists received these

162 scores of the patient at the first visit, so that they could evaluate whether the patient had had any

163 progression on that scale. Each neurologist was then asked to reach a final clinical diagnosis of

164 the parkinsonian syndrome. One investigator compared these scores and when there was no

165 agreement, the two neurologists were asked to discuss these patients using their notes, in an

166 effort to reach agreement on the final diagnosis (Bouwmans et al. 2013). 


\section{Statistics}

170

171 SPSS 21.0 for Windows was used for the statistical analysis. Comparing categorical variables

172 was done by chi-square test. The two-sample t-test was used for comparing continuous variables.

173 Before performing a post-hoc test, we used the homogeneity of variances to decide which post-

174 hoc test was suitable. When showing a good homogeneity, we chose the Bonferroni or Tamhane

175 T2 test for further analyses, otherwise. P values of $<0.05$ were considered significant.

176

177 


\section{Results}

179

180 Patient characteristics

181 We allocated patients who were eventually diagnosed with essential tremor (ET) in the group of

182 parkinsonism, because of the pathophysiologic resemblance with PD (Adler et al. 2011; Fekete

183 \& Jankovic 2011; Louis \& Ottman 2013; Shahed \& Jankovic 2007; Tan et al. 2008). We had an

184 insufficient bone window in $18.7 \%$ of our patients. This is in line with earlier studies that report

185 an insufficient bone window in 10 to $20 \%$ of participants, or even up to $59 \%$ of women over 60

186 years (Okawa et al. 2007; Walter et al. 2007a).

187

188 Eventually, we were able to obtain interpretable TCS images of the SN in 126 patients with

189 HAMD rating scale scores. We did TCS of the RN in only one of the two hospitals, so in the end

190 we had 80 patients with RN TCS images. Only later on in the study did we start measuring the

191 width of the third ventricle (An amendment to the study protocol was made), so this echo feature

192 was available for only 57 of the 126 patients.

193

194 At follow-up, 72 (57\%) patients were clinically diagnosed with PD. 19 (15\%) patients had

195 atypical parkinsonian syndromes (APS), such as multiple system atrophy (MSA), progressive

196 supranuclear palsy (PSP), Lewy body dementia (LBD) and corticobasal degeneration (CBD).

197 Nineteen (15\%) patients had vascular parkinsonism (VP) and 16 (13\%) were diagnosed with ET

198 (See table 1). The subgroups differed significantly on a number of variables, with PD patients

199 being younger and having higher (worse) UPDRS scores (Table 1). The average HAMD scores

200 did not differ between the groups. Sixteen (13\%) of the patients had a HAMD $\geq 11$, indicating 
201 clinically relevant depressive symptoms. The percentage of patients with a hyperechogenic SN

202 and the percentage with hypo-echogenic RN, did not differ between the group with PD and the

203 one with other parkinsonisms. We compared actual HAMD scores between two groups

204 (hyperechogenicity and hypo-echogenicity) with a t-test, but were unable to find a significant

205 association $(\mathrm{SN}+$ mean $4.22, \mathrm{SN}-5.82, \mathrm{t}=1.70, \mathrm{p}=0.92)$. The width of the third ventricle was also

206 not significantly different between the two groups.

207

208 Depression and echo features in PD and other parkinsonisms

209 Nine $(13 \%)$ of the 72 patients with PD had a HAMD $>11$ versus 7 of the 4 patients with other

210 parkinsonisms. There were no differences in the three TCS features between depressed and non-

211 depressed PD patients. In patients with other parkinsonisms we found a significantly higher

212 frequency of hyperechogenic SN in non-depressed patients (See Table 2). There were no

213 significant differences in echo features of $\mathrm{RN}$ and the third ventricle between depressed and non-

214 depressed patients with other parkinsonisms (See figures 1-3).

215

216 


\section{Discussion}

218

219 In this cross-sectional study of 126 early stage parkinsonian patients we did not find any relation

220 between the presence of depressive symptoms and the echogenicity of the SN, RN nor the width

221 of the third ventricle. We found a higher frequency of hyperechogenicity of the SN in the non-

222 depressed patients with other parkinsonisms, but the significance of this remains unclear as it is

223 the result of a posthoc subgroup analysis.

224

225 The major limitation of our study is that it is a secondary analysis of a study that was not

226 powered to detect differences in echogenicity of the $\mathrm{SN}$ and $\mathrm{RN}$, or width of the third ventricle

227 resulting from the severity of depressive symptoms. This resulted in a relatively low proportion

228 of patients with clinically relevant depressive symptoms and subgroup analyses were done with a

229 limited number of depressed patients. Because of that, our results must be seen as exploratory

230 and interpreted with caution. Especially the lack of a significant difference in the proportion of

231 hyperechogenicity of the SN between depressed and non-depressed PD patients may be due to a

232 lack of power. Another limitation is the lack of a formal psychiatric assessment to support a

233 diagnosis of depression based on diagnostic criteria. However, a HAMD score $\geq 11$ is considered

234 a good indicator of clinically relevant depressive symptoms and has been used to screen for

235 depression in several studies (Leentjens et al. 2000; Reijnders et al. 2010; Schrag 2011; Schrag

236 et al. 2007). Finally, a limitation of all these studies is the lack of a gold standard clinical

237 diagnosis. The accepted gold standard is postmortem neuropathological examination, which is

238 shown to differ substantially even from movement disorder specialists' diagnoses (Joutsa et al.

239 2014). However, for practical reasons, clinical examination after several years is a reasonable

240 surrogate. Another limitation is the 2 years' follow-up. While longer follow-up is preferable, 2 
241 years is enough to assess the three most important temporal diagnostic criteria for Parkinson's

242 disease (Hughes et al. 1992).

245 As the RN are thought to play a role in the pathogenesis of depression, investigators have used

246 TCS to visualize these structures in depressed patients. They found that depressed (Becker et al.

247 1995; Becker et al. 2001; Becker et al. 1994; Walter et al. 2007c; Walter et al. 2007d) and

248 bipolar patients (Krogias et al. 2011) have a reduced echogenicity of the RN compared to healthy

249 controls. Others reported that PD patients with depression have lower RN echogenicity

250 compared to non-depressed PD patients and healthy control subjects (Becker et al. 1997; Berg et

251 al. 1999b; Cho et al. 2011; Stankovic et al. 2015; Walter et al. 2007b; Zhang et al. 2015)

252 suggesting that hypo-echogenicity of the RN may be a sign of (preclinical) dysfunction of the

253 limbic system. However, in our study we could not confirm these findings.

254

255 There are essential differences between the above five studies that did find an association and

256 ours that did not. First, the definition of depression was different. Some of the previous studies

257 used DSM-III and DSM-IV criteria to diagnose depressive disorder (not specified if this was

258 done by a board-certified psychiatrist), and others used the Hamilton Depression and

259 Montgomery-Asberg Depression Rating Scale, with varying cut-offs. Secondly, patient selection

260 was different. We followed a prospective study in which consecutive patients were enrolled. The

261 other studies, with the exception of Stankovic's, did not describe how patients were recruited,

262 and it is not clear whether this might have biased results (Colditz 2010). Another difference is

263 the disease duration of the included patients: with the exception of Cho's study, all studies 
264 included patients with a longer disease duration, up to $17 \mathrm{yrs}$. even (Berg et al. 1999a). This is

265 possibly also reflected in the high percentages of patients with clinically relevant depressive

266 symptoms in these studies: $43-47 \%$, while this prevalence is generally assumed to be around

$26735 \%$ (Reijnders et al. 2008). This approach is useful in pilot experiments, but when one wants to

268 assess diagnostic accuracy of a technique it is preferable to include patients who have not yet

269 been definitely diagnosed (Bachmann et al. 2009). The inclusion of early, not yet diagnosed

270 patients in our study may explain the low percentage $(13 \%)$ of patients with clinically relevant

271 symptoms.

272

273 Some studies have suggested a relation between the width of the third ventricle and the presence

274 of depression (Krogias et al. 2011), as enlargement of the third ventricle may be a reflection of

275 the atrophy of the surrounding structures (Hendrie \& Pickles 2010). We were also not able to

276 confirm these findings.

277

278 In a meta-analysis we did on TCS in parkinsonian syndromes we found that in 7 retrospective

279 studies a decreased echo-intensity of the $\mathrm{RN}$ was found more often in depressed (46\%) than in

280 non-depressed IPD patients (16\%). Our present study does not accord with that observation. We

281 hypothesize that one of the main reasons for this is publication bias, where negative studies on

282 TCS tend not to be published. We did not formally test that in our meta-analysis, but another

283 example is our recent negative study on the accuracy of TCS to diagnose IPD. Many studies

284 (Becker et al. 1995b; Berg et al. 2001; Gaenslen et al. 2008; Huang et al. 2007; Kim et al. 2007;

285 Mehnert et al. 2010; Ressner et al. 2007; Spiegel et al. 2006; Walter et al. 2002) found a striking

286 association between a hyperechogenic SN and the diagnosis of PD, but in a carefully designed 
287 and executed diagnostic accuracy study we could not confirm these results (Bouwmans et al.

288 2013).

289

290 In conclusion, in early stage parkinsonian patients we did not find any relation between the

291 presence of depressive symptoms and the echogenicity of the SN, RN nor the width of the third

292 ventricle. At present this technique has limited diagnostic value to diagnose or predict depression

293 in parkinsonian patients.

\section{References:}

300

301

302

303

304

305

306

307

308

309

310

311

312

313

314

315

316

317

318

Bachmann LM, ter Riet G, Weber WE, and Kessels AG. 2009. Multivariable adjustments counteract spectrum and test review bias in accuracy studies. J Clin Epidemiol 62:357361 e352. 10.1016/j.jclinepi.2008.02.007

Becker G, Becker T, Struck M, Lindner A, Burzer K, Retz W, Bogdahn U, and Beckmann H. 1995. Reduced echogenicity of brainstem raphe specific to unipolar depression: a transcranial color-coded real-time sonography study. Biol Psychiatry 38:180-184. 00063223(94)00263-3 [pii]

10.1016/0006-3223(94)00263-3

Becker G, Berg D, Lesch KP, and Becker T. 2001. Basal limbic system alteration in major depression: a hypothesis supported by transcranial sonography and MRI findings. Int $J$ Neuropsychopharmacol 4:21-31. doi:10.1017/S1461145701002164

S1461145701002164 [pii]

Becker G, Struck M, Bogdahn U, and Becker T. 1994. Echogenicity of the brainstem raphe in patients with major depression. Psychiatry Res 55:75-84.

Becker T, Becker G, Seufert J, Hofmann E, Lange KW, Naumann M, Lindner A, Reichmann H, Riederer P, Beckmann H, and Reiners K. 1997. Parkinson's disease and depression: evidence for an alteration of the basal limbic system detected by transcranial sonography. J Neurol Neurosurg Psychiatry 63:590-596. 
319 Berg D, Becker G, Zeiler B, Tucha O, Hofmann E, Preier M, Benz P, Jost W, Reiners K, and

320

321

322

323

324

325

326

327

328

329

330

331

332

333

334

335

336

337

338

339

340

341

342

343

344

345

346

347

348

349

350

351

352

353

354

355

356

357

358

359

360

361

362

363

Lange KW. 1999a. Vulnerability of the nigrostriatal system as detected by transcranial ultrasound. Neurology 53:1026-1031.

Berg D, Supprian T, Hofmann E, Zeiler B, Jager A, Lange KW, Reiners K, Becker T, and Becker G. 1999b. Depression in Parkinson's disease: brainstem midline alteration on transcranial sonography and magnetic resonance imaging. $J$ Neurol 246:1186-1193.

Bouwmans AE, Vlaar AM, Mess WH, Kessels A, and Weber WE. 2013. Specificity and sensitivity of transcranial sonography of the substantia nigra in the diagnosis of Parkinson's disease: prospective cohort study in 196 patients. BMJ Open 3. 10.1136/bmjopen-2013-002613

Bouwmans AE, and Weber WE. 2012. Neurologists' diagnostic accuracy of depression and cognitive problems in patients with parkinsonism. BMC Neurol 12:37. 1471-2377-12-37 [pii]

10.1186/1471-2377-12-37

Chagas MH, Linares IM, Garcia GJ, Hallak JE, Tumas V, and Crippa JA. 2013. Neuroimaging of depression in Parkinson's disease: a review. Int Psychogeriatr 25:1953-1961. S1041610213001427 [pii]

$10.1017 / \mathrm{S} 1041610213001427$

Cho JW, Baik JS, and Lee MS. 2011. Mesencephalic midline change on transcranial sonography in early Parkinson's disease patients with depression. J Neurol Sci 310:50-52. S0022$510 \mathrm{X}(11) 00477-1$ [pii]

10.1016/j.jns.2011.07.055

Colditz GA. 2010. Overview of the epidemiology methods and applications: strengths and limitations of observational study designs. Crit Rev Food Sci Nutr 50 Suppl 1:10-12. 10.1080/10408398.2010.526838

Gilman S, Wenning GK, Low PA, Brooks DJ, Mathias CJ, Trojanowski JQ, Wood NW, Colosimo C, Durr A, Fowler CJ, Kaufmann H, Klockgether T, Lees A, Poewe W, Quinn N, Revesz T, Robertson D, Sandroni P, Seppi K, and Vidailhet M. 2008. Second consensus statement on the diagnosis of multiple system atrophy. Neurology 71:670-676. $71 / 9 / 670$ [pii]

10.1212/01.wnl.0000324625.00404.15

Hendrie CA, and Pickles AR. 2010. Depression as an evolutionary adaptation: anatomical organisation around the third ventricle. Med Hypotheses 74:735-740. S03069877(09)00705-1 [pii]

10.1016/j.mehy.2009.10.026

Hughes AJ, Daniel SE, Kilford L, and Lees AJ. 1992. Accuracy of clinical diagnosis of idiopathic Parkinson's disease: a clinico-pathological study of 100 cases. J Neurol Neurosurg Psychiatry 55:181-184.

Joutsa J, Gardberg M, Roytta M, and Kaasinen V. 2014. Diagnostic accuracy of parkinsonism syndromes by general neurologists. Parkinsonism Relat Disord 20:840-844. 10.1016/j.parkreldis.2014.04.019

Kostic VS, and Filippi M. 2011. Neuroanatomical correlates of depression and apathy in Parkinson's disease: magnetic resonance imaging studies. J Neurol Sci 310:61-63. S0022$510 \mathrm{X}(11) 00298-\mathrm{X}$ [pii]

10.1016/j.jns.2011.05.036

PeerJ reviewing PDF | (2015:09:6674:2:0:NEW 11 Apr 2016) 
364 Krogias C, Hoffmann K, Eyding J, Scheele D, Norra C, Gold R, Juckel G, and Assion HJ. 2011.

365

366

367

368

369

370

371

372

373

374

375

376

377

378

379

380

381

382

383

384

385

386

387

388

389

390

391

392

393

394

395

396

397

398

399

400

401

402

403

404

405

406

407

408

409

Evaluation of basal ganglia, brainstem raphe and ventricles in bipolar disorder by transcranial sonography. Psychiatry Res 194:190-197. S0925-4927(11)00122-3 [pii]

10.1016/j.pscychresns.2011.04.002

Leentjens AF. 2004. Depression in Parkinson's disease: conceptual issues and clinical challenges. J Geriatr Psychiatry Neurol 17:120-126. 10.1177/0891988704267456

Leentjens AF, Verhey FR, Lousberg R, Spitsbergen H, and Wilmink FW. 2000. The validity of the Hamilton and Montgomery-Asberg depression rating scales as screening and diagnostic tools for depression in Parkinson's disease. Int J Geriatr Psychiatry 15:644649. 10.1002/1099-1166(200007)15:7<644::AID-GPS167>3.0.CO;2-L [pii]

Litvan I, Agid Y, Calne D, Campbell G, Dubois B, Duvoisin RC, Goetz CG, Golbe LI, Grafman J, Growdon JH, Hallett M, Jankovic J, Quinn NP, Tolosa E, and Zee DS. 1996. Clinical research criteria for the diagnosis of progressive supranuclear palsy (Steele-RichardsonOlszewski syndrome): report of the NINDS-SPSP international workshop. Neurology 47:1-9.

Litvan I, Grimes DA, Lang AE, Jankovic J, McKee A, Verny M, Jellinger K, Chaudhuri KR, and Pearce RK. 1999. Clinical features differentiating patients with postmortem confirmed progressive supranuclear palsy and corticobasal degeneration. J Neurol 246 Suppl 2:II15. 9246S001.415 [pii]

McKeith IG, Dickson DW, Lowe J, Emre M, O'Brien JT, Feldman H, Cummings J, Duda JE, Lippa C, Perry EK, Aarsland D, Arai H, Ballard CG, Boeve B, Burn DJ, Costa D, Del Ser T, Dubois B, Galasko D, Gauthier S, Goetz CG, Gomez-Tortosa E, Halliday G, Hansen LA, Hardy J, Iwatsubo T, Kalaria RN, Kaufer D, Kenny RA, Korczyn A, Kosaka K, Lee VM, Lees A, Litvan I, Londos E, Lopez OL, Minoshima S, Mizuno Y, Molina JA, Mukaetova-Ladinska EB, Pasquier F, Perry RH, Schulz JB, Trojanowski JQ, and Yamada M. 2005. Diagnosis and management of dementia with Lewy bodies: third report of the DLB Consortium. Neurology 65:1863-1872. 01.wnl.0000187889.17253.b1 [pii] 10.1212/01.wnl.0000187889.17253.b1

Movement Disorder Society Task Force on Rating Scales for Parkinson's D. 2003. The Unified Parkinson's Disease Rating Scale (UPDRS): status and recommendations. Mov Disord 18:738-750. 10.1002/mds.10473

Okawa M, Miwa H, Kajimoto Y, Hama K, Morita S, Nakanishi I, and Kondo T. 2007. Transcranial sonography of the substantia nigra in Japanese patients with Parkinson's disease or atypical parkinsonism: clinical potential and limitations. Intern Med 46:15271531. JST.JSTAGE/internalmedicine/46.0271 [pii]

Palhagen SE, Carlsson M, Curman E, Walinder J, and Granerus AK. 2008. Depressive illness in Parkinson's disease--indication of a more advanced and widespread neurodegenerative process? Acta Neurol Scand 117:295-304. ANE986 [pii]

10.1111/j.1600-0404.2007.00986.x

Poewe W, and Luginger E. 1999. Depression in Parkinson's disease: impediments to recognition and treatment options. Neurology 52:S2-6.

Pringsheim T, Jette N, Frolkis A, and Steeves TD. 2014. The prevalence of Parkinson's disease: a systematic review and meta-analysis. Mov Disord 29:1583-1590. 10.1002/mds.25945

Reijnders JS, Ehrt U, Weber WE, Aarsland D, and Leentjens AF. 2008. A systematic review of prevalence studies of depression in Parkinson's disease. Mov Disord 23:183-189; quiz 313. $10.1002 / \mathrm{mds} .21803$

PeerJ reviewing PDF | (2015:09:6674:2:0:NEW 11 Apr 2016) 
410 Reijnders JS, Lousberg R, and Leentjens AF. 2010. Assessment of depression in Parkinson's

411

412

413

414

415

416

417

418

419

420

421

422

423

424

425

426

427

428

429

430

431

432

433

434

435

436

437

438

439

440

441

442

443

444

445

446

447

448

449

450

451

452

453 disease: the contribution of somatic symptoms to the clinimetric performance of the Hamilton and Montgomery-Asberg rating scales. J Psychosom Res 68:561-565. S00223999(09)00416-4 [pii]

10.1016/j.jpsychores.2009.10.006

Schrag A. 2006. Quality of life and depression in Parkinson's disease. J Neurol Sci 248:151-157. S0022-510X(06)00226-7 [pii]

10.1016/j.jns.2006.05.030

Schrag A. 2011. Apathy and depression scales in Parkinson's disease: are they good enough? $J$ Neurol Sci 310:216-219. S0022-510X(11)00307-8 [pii]

10.1016/j.jns.2011.05.045

Schrag A, Barone P, Brown RG, Leentjens AF, McDonald WM, Starkstein S, Weintraub D, Poewe W, Rascol O, Sampaio C, Stebbins GT, and Goetz CG. 2007. Depression rating scales in Parkinson's disease: critique and recommendations. Mov Disord 22:1077-1092. 10.1002/mds. 21333

Schrag A, Sheikh S, Quinn NP, Lees AJ, Selai C, Mathias C, Litvan I, Lang AE, Bower JH, Burn DJ, Low P, and Jahanshahi M. 2010. A comparison of depression, anxiety, and health status in patients with progressive supranuclear palsy and multiple system atrophy. Mov Disord 25:1077-1081. 10.1002/mds.22794

Shulman LM, Taback RL, Rabinstein AA, and Weiner WJ. 2002. Non-recognition of depression and other non-motor symptoms in Parkinson's disease. Parkinsonism Relat Disord 8:193197. S1353802001000153 [pii]

Stankovic I, Stefanova E, Ziropadja L, Mijajlovic M, Pavlovic A, and Kostic VS. 2015. Transcranial midbrain sonography and depressive symptoms in patients with Parkinson's disease. J Neurol 262:689-695. 10.1007/s00415-014-7624-0

Vlaar AM, Bouwmans A, Mess WH, Tromp SC, and Weber WE. 2009. Transcranial duplex in the differential diagnosis of parkinsonian syndromes: a systematic review. $J$ Neurol 256:530-538. 10.1007/s00415-009-0143-8

Vlaar AM, Bouwmans AE, van Kroonenburgh MJ, Mess WH, Tromp SC, Wuisman PG, Kessels AG, Winogrodzka A, and Weber WE. 2007. Protocol of a prospective study on the diagnostic value of transcranial duplex scanning of the substantia nigra in patients with parkinsonian symptoms. BMC Neurol 7:28. 1471-2377-7-28 [pii]

10.1186/1471-2377-7-28

Walter U, Behnke S, Eyding J, Niehaus L, Postert T, Seidel G, and Berg D. 2007a. Transcranial brain parenchyma sonography in movement disorders: state of the art. Ultrasound Med Biol 33:15-25. S0301-5629(06)01749-2 [pii]

10.1016/j.ultrasmedbio.2006.07.021

Walter U, Dressler D, Wolters A, Wittstock M, and Benecke R. 2007b. Transcranial brain sonography findings in clinical subgroups of idiopathic Parkinson's disease. Mov Disord 22:48-54. 10.1002/mds.21197

Walter U, Hoeppner J, Prudente-Morrissey L, Horowski S, Herpertz SC, and Benecke R. 2007c. Parkinson's disease-like midbrain sonography abnormalities are frequent in depressive disorders. Brain 130:1799-1807. awm017 [pii]

10.1093/brain/awm017 
454 Walter U, Prudente-Morrissey L, Herpertz SC, Benecke R, and Hoeppner J. 2007d. Relationship 455 of brainstem raphe echogenicity and clinical findings in depressive states. Psychiatry Res 456 457 155:67-73. S0925-4927(06)00225-3 [pii]

10.1016/j.pscychresns.2006.12.001

Walter U, Skoloudik D, and Berg D. 2010. Transcranial sonography findings related to nonmotor features of Parkinson's disease. J Neurol Sci 289:123-127. S0022-510X(09)00783$460 \quad 7$ [pii]

$461 \quad$ 10.1016/j.jns.2009.08.027

462 Zhang YC, Hu H, Luo WF, Sheng YJ, Chen XF, Mao CJ, Xiong KP, Yu LF, Zhang Y, and Liu

463

464

465 CF. 2015. Alteration of brainstem raphe measured by transcranial sonography in

466 depression patients with or without Parkinson's disease. Neurol Sci. 10.1007/s10072-0152350-7 
Table $\mathbf{1}$ (on next page)

Patient characteristics divided by final diagnoses

PD = Parkinson's disease; UPDRS = Unified Parkinson's Disease Rating Scale;

DP+= depression present/ having a total score of the HAMD of 11 or more, SN+= hyperechogenic substantia nigra; $\mathbf{R N}-=$ hypo-echogenic raphe nuclei [b] 
1 Table 1. Patient characteristics divided by final diagnoses:

\begin{tabular}{|c|c|c|c|}
\hline & $\begin{array}{l}P D \\
(n=72)\end{array}$ & $\begin{array}{l}\text { Other } \\
\text { parkinsonisms } \\
(n=54)\end{array}$ & $P$ value \\
\hline $\begin{array}{l}\text { Age, years } \\
(\mathrm{SD}, \mathrm{Cl})\end{array}$ & $\begin{array}{l}68.6 \\
(9.2,66.43- \\
70.76)\end{array}$ & $\begin{array}{l}72.2 \\
(9.3,69.69- \\
74.79)\end{array}$ & 0.03 \\
\hline $\begin{array}{l}\text { disease } \\
\text { duration } \\
\text {,months (SD, } \\
\mathrm{Cl} \text { ) }\end{array}$ & $\begin{array}{l}30.1 \\
(47.1,19.01- \\
41.16)\end{array}$ & $\begin{array}{l}41.7 \\
(41.4,30.42- \\
53.02)\end{array}$ & 0.15 \\
\hline $\begin{array}{l}\text { UPDRS total } \\
\text { score,mean } \\
\text { (SD, Cl) }\end{array}$ & $\begin{array}{l}24.5 \\
(10.6,21.99- \\
27.00)\end{array}$ & $\begin{array}{l}10.6 \\
(15.6,24.94- \\
33.60)\end{array}$ & 0.06 \\
\hline $\begin{array}{l}\text { UPDRS motor } \\
\text { score,mean } \\
(\mathrm{SD}, \mathrm{Cl})\end{array}$ & $\begin{array}{l}13.2 \\
(5.7,11.82- \\
14.55)\end{array}$ & $\begin{array}{l}15.0 \\
(7.8,12.85- \\
17.15)\end{array}$ & 0.16 \\
\hline $\begin{array}{l}\text { HAMD, mean, } \\
(\mathrm{SD}, \mathrm{CI})\end{array}$ & $\begin{array}{l}4.6 \\
(5.5,3.33- \\
5.92)\end{array}$ & $\begin{array}{l}5.8 \\
(5.5,4.28- \\
7.28)\end{array}$ & 0.25 \\
\hline DP+ \% & 12.5 & 13.0 & 0.94 \\
\hline $\mathrm{SN}+\%$ & 43.06 & 44.4 & 0.88 \\
\hline RN- \% & 21.7 & 17.7 & 0.65 \\
\hline $\begin{array}{l}\text { Third } \\
\text { ventricle } \\
\text { width, } \mathrm{mm} \\
\text { (SD, } \mathrm{Cl})\end{array}$ & $\begin{array}{l}5.4 \\
(2.10,4.62- \\
6.17)\end{array}$ & $\begin{array}{l}5.3 \\
(2.38,4.36- \\
6.28)\end{array}$ & 0.90 \\
\hline
\end{tabular}

$2 \mathrm{PD}=$ Parkinson's disease; UPDRS = Unified Parkinson's Disease Rating Scale; $\mathrm{DP}+=$ depression present/

3 having a total score of the HAMD of 11 or more, $\mathrm{SN}+=$ hyperechogenic substantia nigra; $\mathrm{RN}-=$ hypo-

4 echogenic raphe nuclei, $\mathrm{SD}=$ standard deviation, $\mathrm{Cl}=$ confidence interval 
Table 2 (on next page)

Patient characteristics divided by final diagnosis

PD = Parkinson's disease; UPDRS = Unified Parkinson's Disease Rating Scale;

DP+= depression present/ having a total score of the HAMD of 11 or more, SN+= hyperechogenic substantia nigra; $\mathrm{RN}$-= hypo-echogenic raphe nuclei 
1 Table 2. Patient characteristics divided by presence or absence of depression:

\begin{tabular}{|c|c|c|c|c|c|c|}
\hline & $\begin{array}{l}\text { PD and } \\
\text { absence } \\
\text { depression } \\
(n=63)\end{array}$ & $\begin{array}{l}\text { PD and } \\
\text { presence } \\
\text { depression }\end{array}$ & $\begin{array}{l}P \\
\text { value }\end{array}$ & $\begin{array}{l}\text { Other } \\
\text { parkinsonisms } \\
\text { and absence } \\
\text { depression } \\
(n=47)\end{array}$ & $\begin{array}{l}\text { Other } \\
\text { parkinsonisms } \\
\text { and presence } \\
\text { depression } \\
(n=7)\end{array}$ & $\begin{array}{l}P \\
\text { value }\end{array}$ \\
\hline $\begin{array}{l}\text { Mean } \\
\text { age,years } \\
(\mathrm{SD}, \mathrm{Cl})\end{array}$ & $\begin{array}{l}69.8 \\
(8.8,67.04- \\
71.50)\end{array}$ & $\begin{array}{l}63.9 \\
(10.9 \\
55.52- \\
72.26)\end{array}$ & 0.10 & $\begin{array}{l}71.9 \\
(9.4,69.18- \\
74.70)\end{array}$ & $\begin{array}{l}74.3 \\
(9.4,65.61- \\
82.96)\end{array}$ & 0.54 \\
\hline $\begin{array}{l}\text { Mean } \\
\text { duration } \\
\text { complaints, } \\
\text { months } \\
(\mathrm{SD}, \mathrm{Cl})\end{array}$ & $\begin{array}{l}32.0 \\
(49.8,19.48- \\
44.59)\end{array}$ & $\begin{array}{l}16.4 \\
(14.6,5.25- \\
27.64)\end{array}$ & 0.36 & $\begin{array}{l}4.7 \\
(42.7,32.21- \\
57.28)\end{array}$ & \begin{tabular}{|l|}
21.4 \\
$(24.8,-1.46-$ \\
$44.32)$
\end{tabular} & 0.17 \\
\hline $\begin{array}{l}\text { UPDRS total } \\
\text { score, mean } \\
\text { (SD, CI) }\end{array}$ & $\begin{array}{l}24.2 \\
(10.3,21.56- \\
26.80)\end{array}$ & $\begin{array}{l}26.7 \\
(12.8 \\
16.85- \\
36.49)\end{array}$ & 0.82 & $\begin{array}{l}27.5 \\
(14.3,23.26- \\
31.74)\end{array}$ & \begin{tabular}{|l|}
42.8 \\
$(19.7,22.19-$ \\
$63.48)$
\end{tabular} & 0.02 \\
\hline $\begin{array}{l}\text { UPDRS } \\
\text { motor } \\
\text { score,mean } \\
(\mathrm{SD}, \mathrm{CI})\end{array}$ & $\begin{array}{l}13.3 \\
(5.4,11.86- \\
14.63)\end{array}$ & $\begin{array}{l}12.8 \\
(8.0,6.61- \\
18.95)\end{array}$ & 0.82 & $\begin{array}{l}14.3 \\
(7.5,12.06- \\
16.45)\end{array}$ & $\begin{array}{l}20.8 \\
(8.4,12.02- \\
29.65)\end{array}$ & 0.05 \\
\hline $\begin{array}{l}\text { HAMD, } \\
\text { mean } \\
(\mathrm{SD}, \mathrm{CI})\end{array}$ & $\begin{array}{l}3.0 \\
(3.2,2.18- \\
3.79)\end{array}$ & $\begin{array}{l}16.1 \\
(4.4,12.75- \\
19.47)\end{array}$ & 0.00 & $\begin{array}{l}4.1 \\
(2.7,3.31- \\
4.90)\end{array}$ & $\begin{array}{l}17.0 \\
(6.5,11.01- \\
22.99)\end{array}$ & 0.00 \\
\hline $\begin{array}{l}\mathrm{SN}+\% \\
\text { (Cl) }\end{array}$ & $\begin{array}{l}46.0 \\
(7.1,42.9)\end{array}$ & 22.2 & 0.18 & 51.1 & 0 & 0.01 \\
\hline $\begin{array}{l}\text { RN- \% } \\
\text { (Cl) }\end{array}$ & $\begin{array}{l}21.1 \\
(-28.7,36.6)\end{array}$ & 25.0 & 0.81 & 20.0 & 0 & 0.32 \\
\hline $\begin{array}{l}\text { Third } \\
\text { ventricle }\end{array}$ & 5.4 & 5.5 & 0.84 & 5.3 & 5.3 & 0.95 \\
\hline
\end{tabular}




\begin{tabular}{|l|l|l|l|l|l|l|}
\hline $\begin{array}{l}\text { width, mm } \\
\text { (SD, CI) }\end{array}$ & $(1.9,4.57-$ & $(3.0,2.43-$ & & $(2.5,4.22-$ & $(1.7,2.60-$ & \\
& $6.16)$ & $8.63)$ & $6.45)$ & $7.90)$ & \\
\hline
\end{tabular}

$2 \mathrm{SN}+=$ hyperechogenic substantia nigra, $\mathrm{RN}-=$ hypo-echogenic raphe nuclei, $\mathrm{SD}=$ standard deviation, $\mathrm{Cl}=$ 3 confidence interval

4

5 
1

Echogenicity of the substantia nigra in patients referred for parkinsonism, by depression $(\mathrm{N}=16$ with $\operatorname{HamD}>10, \mathrm{n}=110$ with HamD $<11$ ) and by final diagnosis (IPD, $\mathrm{n}=72$; other, $n=54)$

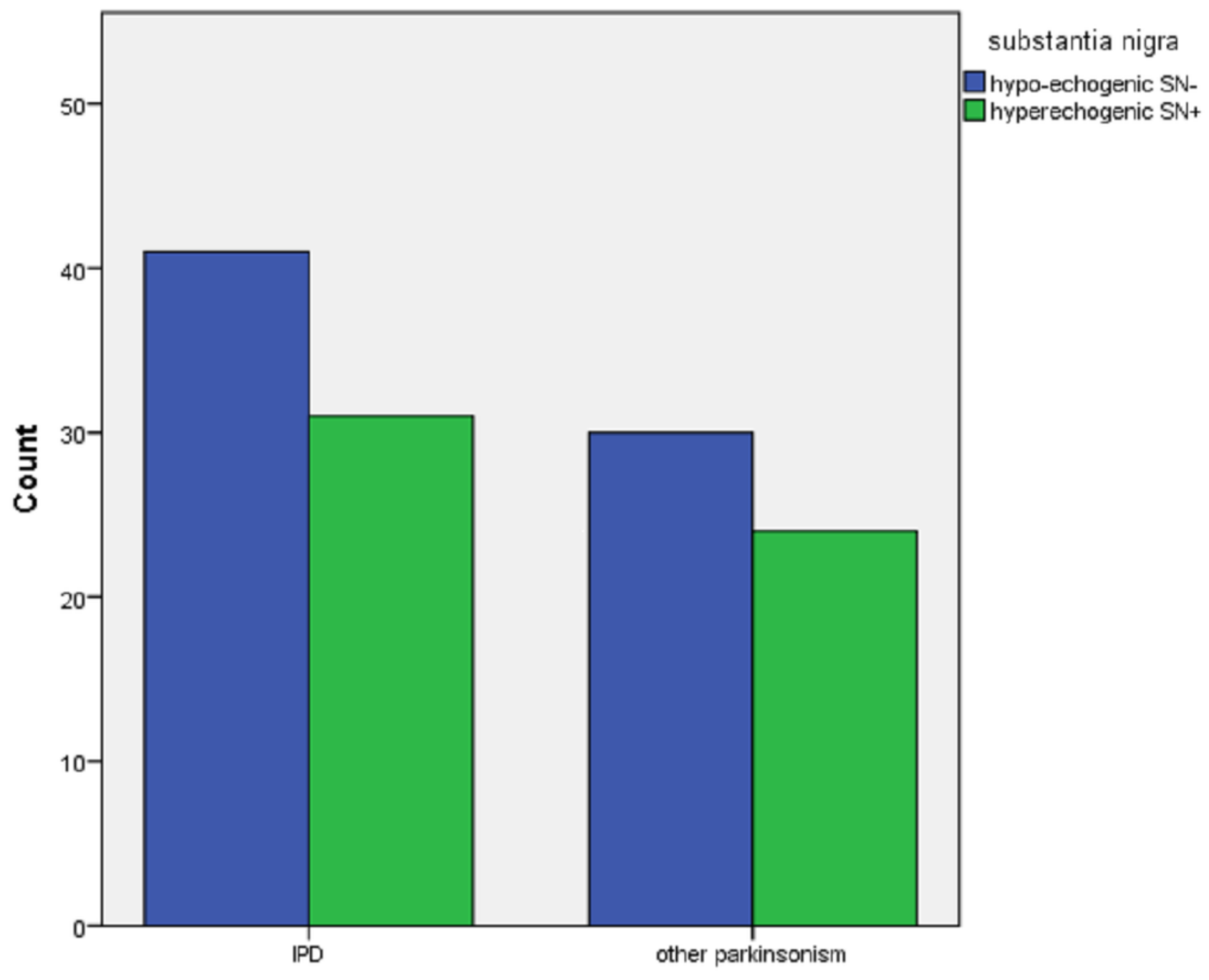

final diagnosis 
2

Echogenicity of the raphe nuclei in patients referred for parkinsonism by depression $(\mathrm{n}=12$ with $\operatorname{HamD}>10, \mathrm{n}=68$ with $\operatorname{HamD}<11$ ), and by final diagnosis (IPD, $n=46$; other $(n=34)$

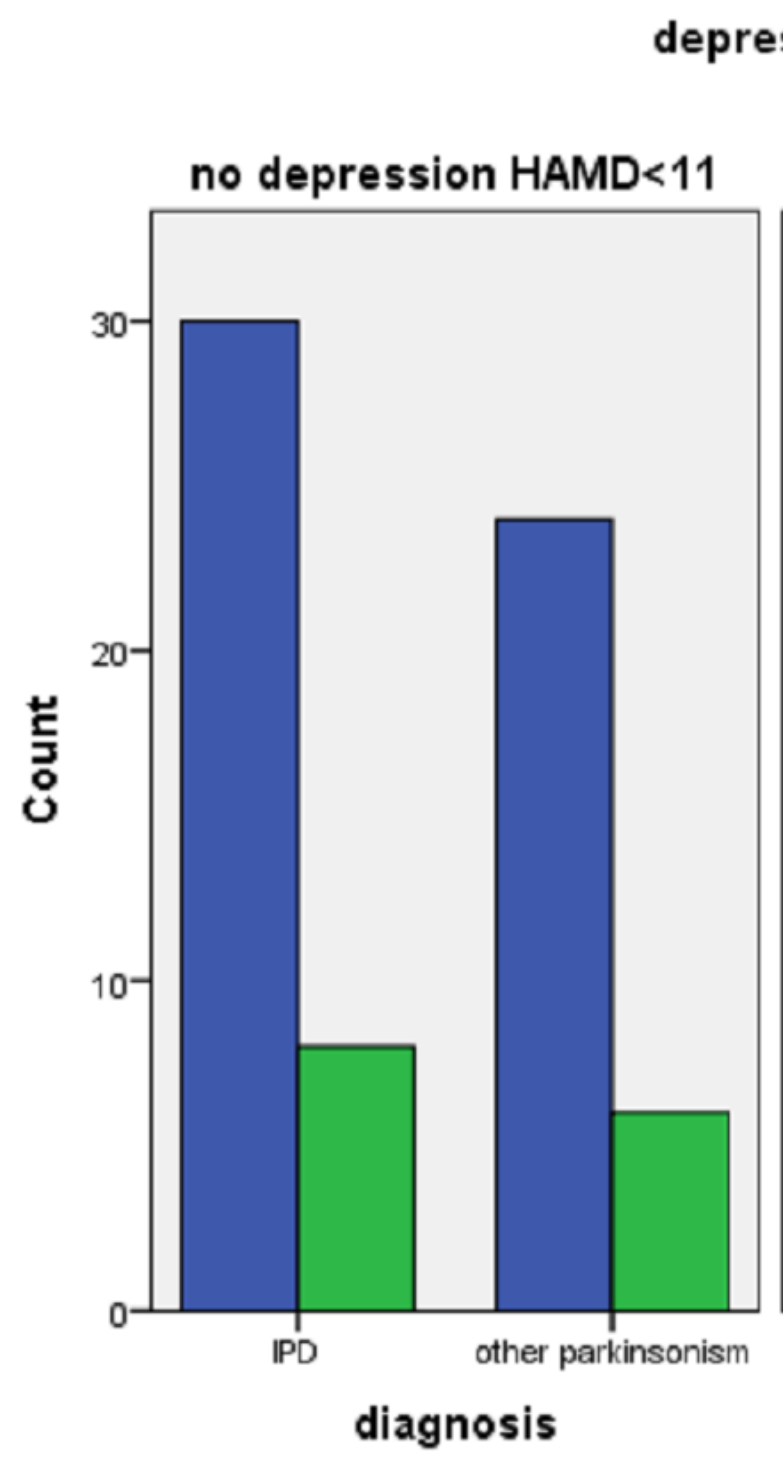

raphe nuclei

Ehyperechogenic RN+ presence depression $\square$ hypoechogenic RN-

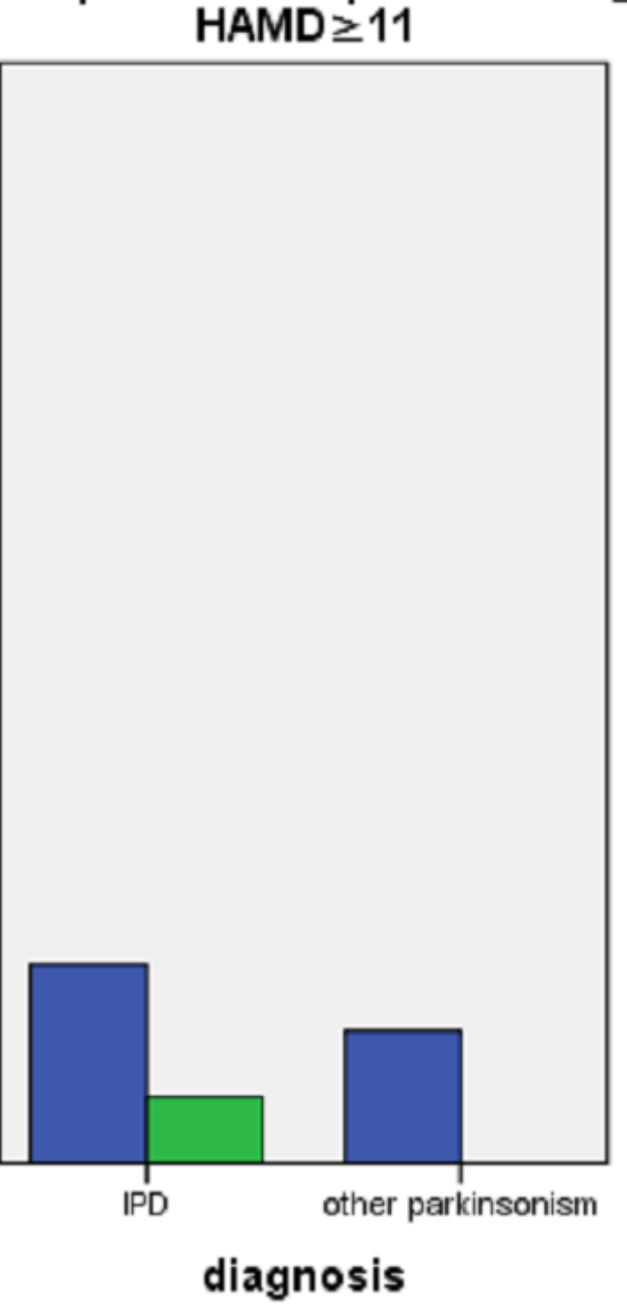


3

Division of width of third ventricle between patients with $(n=10)$ and without a depression $(n=47)$ divided by diagnosis IPD $(n=31)$ and other parkinsonisms $(n=26)$

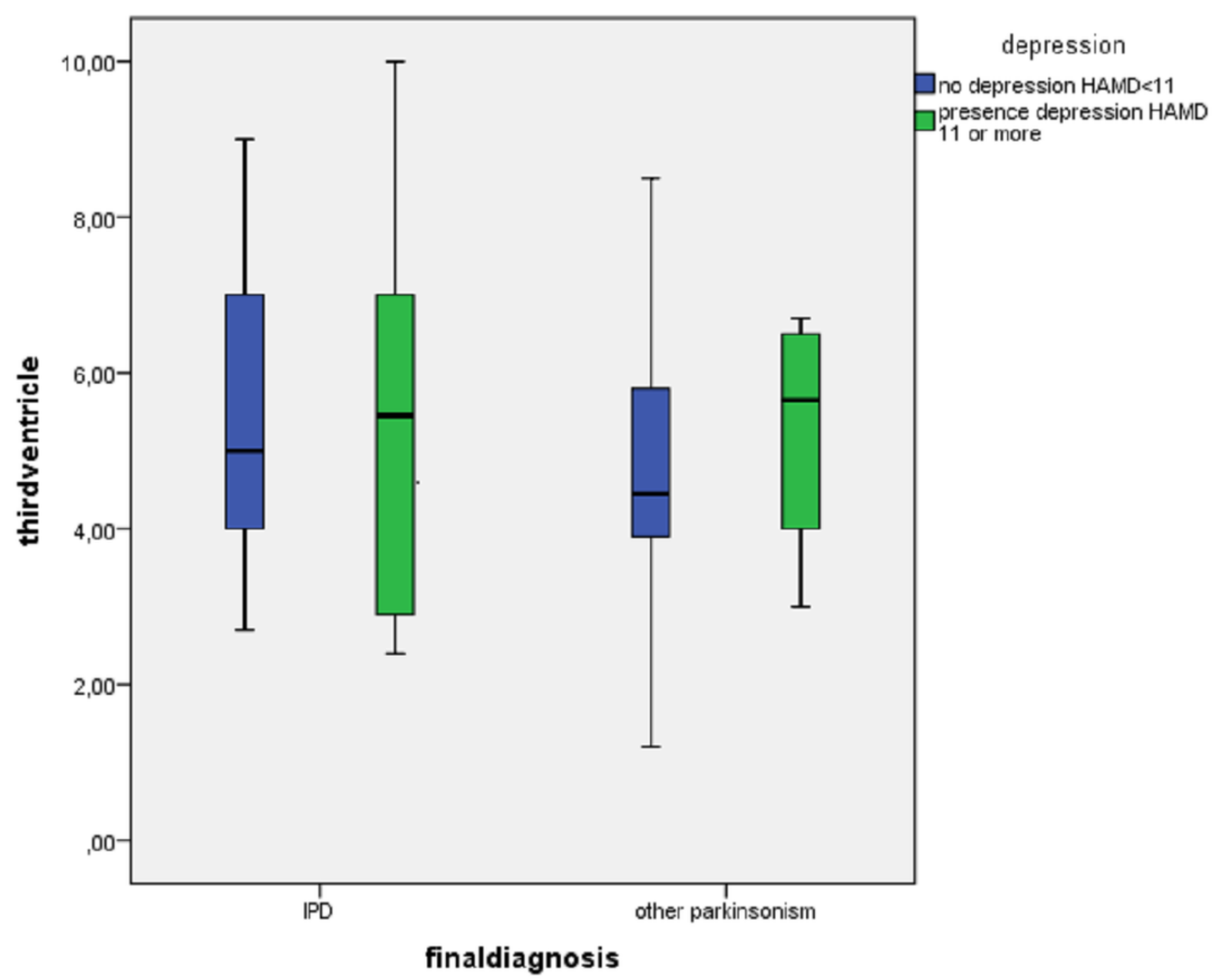

